\title{
A Software-Defined Radio System for Backscatter Sensor Networks
}

\author{
Giovanni Vannucci, Senior Member, IEEE, Aggelos Bletsas, Member, IEEE, and Darren Leigh, Member, IEEE
}

\begin{abstract}
Backscatter radio is proposed for sensor networks. In that way, the transmitter for each sensor is simplified to a transistor connected to an antenna and therefore, the cost for each sensor's communicator becomes negligible, while energy used for wireless communication per sensor is minimized. A software-defined transceiver is built to transmit a carrier, receive the reflections from various sensors and extract their transmitted messages. This work presents a thorough model of the backscatter radio link, the system architecture and a set of data extraction techniques for each sensor's information, testing in practice a sensor communicating through backscatter at a range of approximately 15 meters indoors, with 5 milliwatt transmission power at 10 bits per second. This work highlights the idiosyncrasies of the backscatter channel and provides a new communication perspective in the fertile area of scalable sensor networks, especially when low bit-rate, ultra-low cost sensors are required.
\end{abstract}

Index Terms-RFID, fading channel, bit error rate, wireless sensor networks.

\section{INTRODUCTION}

$\mathbf{T}$ HE technique of radio backscatter is commonly used in RFID (Radio Frequency IDentification) systems. In a typical application, a RFID tag communicates to a reader (sometimes called an interrogator) its identification number and in some cases additional information stored in its memory. Radio backscatter communication is an attractive solution in such systems because the tag does not need to actively transmit any radio signal; the tag simply reflects a radio signal transmitted by the interrogator and modulates the reflection, by controlling its own reflection coefficient [1]-[3]. This leads to very-low-cost implementations and also very low power requirements in the tag. Indeed, in systems where the distance between tag and interrogator is short (i.e., less than 2-5 $\mathrm{m}$ or so), RFID tags may derive their power from the signal received from the interrogator. Such batteryless tags are known as "passive" and the maximum distance (range) achieved by such systems is determined by the tag's ability to extract enough voltage from the received signal. Even though passive RFID technology has matured since the first work on modulated backscattered signals [1], the range of typical passive RFID systems is limited, at most within a room [4], spanning a

Manuscript received October 6, 2006; revised July 11, 2007; accepted September 4, 2007. The Associate Editor coordinating the review of this paper and approving it for publication was A. Stefanov. This research was supported by Mitsubishi Electric Research Laboratories (MERL), 201 Broadway Avenue, Cambridge, MA 02139 USA. Part of this work was presented at IEEE PIMRC 2007, Athens Greece.

The authors are with Mitsubishi Electric Research Laboratories (MERL), 201 Broadway Avenue, Cambridge, MA 02139 USA (e-mail: \{vannucci, leigh\}@merl.com, aggelos@media.mit.edu).

Digital Object Identifier 10.1109/TWC.2008.060796. variety of applications from classical supply chain monitoring and inventory control to non-traditional applications such as music interfaces [5].

By contrast, RFID systems that require a larger operating distance use tags that are known as "semi-passive" (or "battery-assisted" [6]). Such tags include a battery but, like their passive counterparts, they too use backscatter (rather than active transmission) as the communication mechanism (e.g. [7]). The battery is used only to operate the tag state machine and the backscatter modulator and, as a result, it is possible for such tags to communicate over longer ranges. The maximum range of a semi-passive tag depends on the signalto-noise ratio (SNR) available at the interrogator receiver. This in turn, depends on system parameters such as the power level transmitted by the interrogator, the reflection efficiency of the tag, the SNR efficiency of the modulation scheme, the noise figure of the interrogator receiver, the radio-propagation environment, as well as the bit-rate.

In many RFID implementations, bit rates have to be relatively high because the RFID tag needs to convey its message to the interrogator in a fraction of a second (eg. [8]). By contrast, in sensor networks this limitation does not usually apply. In this work, an architecture for a wireless sensor network is presented, based on radio backscatter communication where the operating range can be as large as 100 meters outdoors. The long range is made possible, in part, by the use of extremely low bit rates between the "tags" (the sensors, in this case) and the "interrogator" (referred to as hub, in this case). Even bit rates as low as 10 bps or less are acceptable because the sensors are at fixed locations, continuously able to backscatter the signal from the hub. In many applications, the sensors need to transmit observed data (e.g. temperature) at intervals of seconds to minutes. Thus, a continuous bit-rate of just a few $\mathrm{Hz}$ is adequate in applications where relatively "slow" environmental changes are monitored.

Utilizing backscatter for sensor networks provides a lowcomplexity and ultra low-cost solution for each sensor transmitter, since the latter could consist of a single RF transistor switch. Apart from the attractive simplicity of the sensor transmitter, backscatter radio simplifies the overall network architecture, since all sensors (tags) unidirectionally transmit towards the interrogator (hub), as opposed to more involved network architectures that rely on multi-hop, self-organized (ad hoc) routing. In [9], a comparison of energy consumption between active RFID (single-hop) and typical wireless sensor network (multi-hop) is attempted, revealing relevant dependencies of communication range and sensor topology on the overall energy budget. In [10], the performance of an ALOHA 
access scheme is modeled and analyzed for an active RFID system where tags only transmit short bursts of their unique identification number.

Despite its attractive conceptual simplicity, backscatter radio for sensor networks as envisioned in this work, must take into account unique constraints (idiosyncracies):

i. Ultra-low complexity sensors: Sensors (tags) in this work meet very stringent requirements of low cost, low power and small size. The transmitter of each sensor consists of a single transistor that switches antenna impedance between two states, while coding and signal conditioning are extremely simple. However, the hub receiver includes more sophisticated signal processing to offset, as much as possible, this limitation.

ii. Unidirectional communication: In many typical RFID applications, tags include a receiver. This allows the tag to detect when it interferes (collides) with other tags and simplifies multiple-access by allowing the interrogator to control tag transmissions. That is the key idea behind Aloha-based or tree-based anti-collision algorithms [11]. By contrast, sensors in this work do not have a receiver and thus, there is no mechanism for coordination or synchronization among them [12]. As such, anti-collision algorithms found in the RFID literature are not an option.

iii. Continuous sensor operation: All sensors in this work continuously backscatter their information. As such, the presented system contrasts with prior systems designed to acquire information from a subset of tags (or a single tag) within a fraction of time and not continuously (see for example pioneering RFID work in [8]).

iv. Ultra-fast varying multi-path: Multi-path is not considered a major problem in typical RFID systems because radio propagation is usually "line of sight" (LOS) and the bit rate is sufficiently high that, if multi-path occurs, it affects received signal strength in an approximately constant fashion over the duration of the message. By contrast, the much lower bit-rate in this work means that phase and amplitude of the received signal may change substantially due to multi-path, over time intervals as short as a couple of bits, even for static (not mobile) sensors. This work provides a detection scheme that is tolerant of such impairments.

Consequently, the contributions of this paper are summarized below:

a. A thorough model of the backscatter wireless channel that highlights its distinctive characteristics and serves as a solid framework for future research in relevant problems such as channel access, signal processing and detection techniques for backscatter radio sensor networks.

b. A concrete set of signal and data extraction techniques for each sensor's information, especially suited to the unidirectional character and fast varying multi-path nature of backscatter channel, as well as the continuous operation of all sensors.

c. A proof-of-concept demonstration of backscatter communication for ultra low-cost sensor networks with a complete hardware and flexible software prototype, using a desktop personal computer (PC).

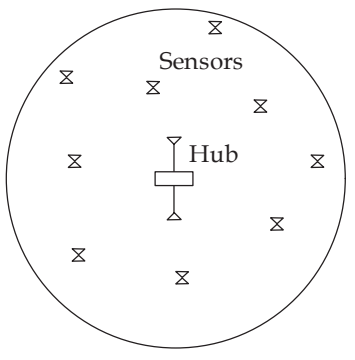

Fig. 1. Layout of a Backscatter-based sensor network. Several low-cost sensors modulate and reflect a carrier transmitted by a central station (hub).

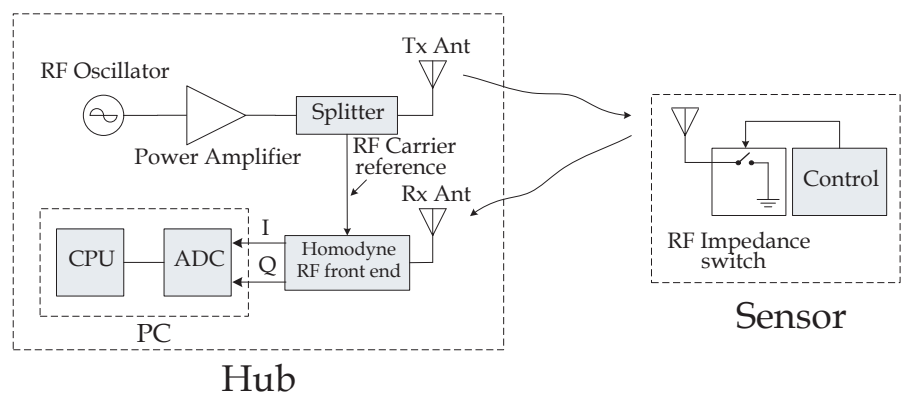

Fig. 2. Backscatter communication between the hub and a single sensor. Notice that the transmitting element at the sensor, is simply a switch made of a transistor.

The presentation is divided in two parts: Section II describes the system architecture, including the basic assumptions and system equations, modulation and access scheme used by all sensors, as well as a summary of the radio prototype. Section III describes data extraction for each sensor, including all techniques used for signal filtering, detection and synchronization. Techniques are evaluated with simulation as well as experimental results from the radio prototype. Finally, conclusion is provided in section IV.

\section{System ARChitecture}

A system of approximately $N=100 \sim 200$ ultra low-cost sensors, located outdoors within approximately $d_{\max }=100$ meters from a central hub, is envisioned (Fig. 1). The sensors monitor and continuously report to the hub, one or more environmental quantities that vary slowly with time. Therefore, the required bit-rate per sensor is limited, on the order of $10 \mathrm{bps} / \mathrm{sensor}$. Such slowly-varying environmental quantities include (but are not limited to) environmental pollutant concentration, humidity or temperature.

\section{A. The Radio Link}

The unmodulated radio-frequency $(\mathrm{RF})$ carrier transmitted by the hub can be written as

$$
s_{\text {hub }}(t)=\sqrt{2 P_{\mathrm{T}}} \exp \left[j\left(\omega_{\mathrm{c}} t+\phi_{\mathrm{c}}\right)\right],
$$

where $P_{\mathrm{T}}, \omega_{\mathrm{c}}, \phi_{\mathrm{c}}$ are the power, angular frequency and phase respectively of the RF carrier transmitted from the hub.

At distance $d_{i}$ between hub and sensor $i$, the signal power has experienced "one-way" propagation link loss $L_{i}$. It is assumed that the hub antenna is mounted at height $h_{\mathrm{T}}$ and 
the sensor antenna at height $h_{\mathrm{R}}$. Taking into account the lineof-sight (LOS) path between hub and sensor antenna, as well as one reflection from the ground, one-way loss $L_{i}$ can be approximated by [13]:

$L_{i}=\frac{\text { received power }}{\text { transmitted power }}= \begin{cases}G_{\mathrm{T}} G_{\mathrm{R}}\left(\lambda / 4 \pi d_{i}\right)^{2}, & \text { if } d_{i}<d_{0} \\ G_{\mathrm{T}} G_{\mathrm{R}}\left(h_{\mathrm{T}} h_{\mathrm{R}} / d_{i}\right)^{2}, & \text { if } d_{i} \geq d_{0}\end{cases}$

where $G_{\mathrm{T}}$ and $G_{\mathrm{R}}$ are the gains of the transmitting (hub) and receiving (sensor) antenna respectively, $\lambda$ is the RF carrier wavelength and $d_{0}$ is given by:

$$
d_{0}=\frac{4 \pi h_{\mathrm{T}} h_{\mathrm{R}}}{\lambda}
$$

Notice that accounting for a single ground reflection, results to the well-studied phenomenon of signal received power being decreased faster than in free space. In this simple scenario, received power drops between the second or fourth power of distance.

At the sensor, the received signal $r_{i}(t)$ is simply reflected back out of the same antenna that received it. Sensor information is modulated onto the reflected signal by varying the sensor's reflection coefficient $\eta_{i}(t)$. Sensor $i$ accomplishes this by controlling a semiconductor device (e.g. a field-effect transistor (FET) or a diode) attached to the antenna (Fig. 2). Accordingly, the reflected signal can be written as:

$$
s_{\mathrm{i}}(t)=\eta_{i}(t) r_{i}(t) .
$$

In practical implementations of backscatter, the reflection coefficient has only two distinct possible states, and backscatter modulation is accomplished by alternating between these two states. Because of that, the reflection coefficient can be written in terms of a binary function of time, $b_{i}(t)$, that only takes the values \pm 1 :

$$
\eta_{i}(t)=\eta_{0}+\eta_{m} b_{i}(t)
$$

where $\eta_{0}$ represents a fixed (unmodulated) complex reflection and $\eta_{m}$ is the amplitude of the modulated component of the reflection. With this notation, the two possible complex reflection coefficients are $\eta_{0}+\eta_{m}$ and $\eta_{0}-\eta_{m}$. The fixed reflection component adds a multitude of fixed reflections from all static scatterers in the environment and as such, it does not contribute in sensor backscatter communication. Consecutively, $\eta_{0}$ is ignored and the above equation is simply written as:

$$
\eta_{i}(t)=\eta_{m} b_{i}(t)
$$

Finally, the backscattered signal travels from sensor $i$ back to the hub and as such, experiences again power loss $L_{i}$. The latter assumes that the hub receive antenna has the same gain and polarization as the hub transmit antenna. Thus, the power of the received signal at the hub, due to backscatter operation at sensor $i$, is given from:

$$
P_{i}^{\text {hub }}=P_{\mathrm{T}} L_{i}^{2} \eta
$$

where $\eta$ is the reflection efficiency of the tag with value dependent on $\eta_{m} \cdot{ }^{1}$ For convenience, it is assumed that all

\footnotetext{
${ }^{1}$ A detailed analysis of optimization parameters related to reflection can be found in [14].
}

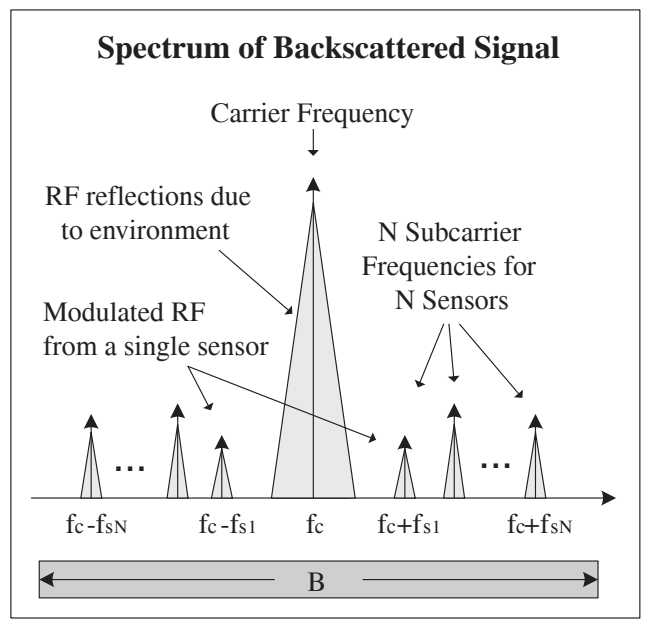

Fig. 3. Spectrum of received signal at the hub receive $(\mathrm{Rx})$ antenna, under the basic assumptions of this work.

sensors are made of the same materials and antenna designs and thus, reflection efficiency $\eta$, as well as $\eta_{m}$, are the same across all sensors. Consecutively, the backscattered signal received at the hub due to sensor $i$, can be written as a complex function of time:

$$
r_{\text {hub }}^{i}(t)=A_{i}(t) b_{i}(t) \exp \left(j \omega_{c} t+\phi_{0}(t)\right),
$$

where $A_{i}(t)$ is the (real) time-varying amplitude and $\phi_{0}(t)$ is the time-varying carrier phase of the received signal, due to backscatter operation of sensor $i$. Notice that both amplitude and carrier phase are functions of time, given the time-varying nature of the wireless channel between hub and sensor $i$. A thorough presentation regarding the time-varying nature of multi-path propagation in wireless channels is beyond the scope of this paper. For our purposes, it is sufficient to note that carrier phase $\phi_{0}$ is time-varying and unknown, in general.

\section{B. The Modulation at Each Sensor}

In each sensor $i$, the function $b_{i}(t)$ generated by the controller is a square wave at a predetermined frequency, referred to as the subcarrier frequency $f_{\mathrm{s} i}$ (or angular subcarrier frequency $\omega_{\mathrm{s} i}$ ) of that sensor. Information from each sensor is modulated onto its unique subcarrier, i.e. different sensors have different subcarrier frequencies. The available sensor subcarrier frequency band is denoted as $\left(f_{\text {sMIN }}, f_{\text {sMAX }}\right)$, with available bandwidth $B=f_{\text {sMAX }}-f_{\text {sMIN }}$ (Fig. 3).

Given that for sensor $i$, the subcarrier waveform $b_{i}(t)$ can only take two values $( \pm 1)$ in the implemented sensors, binary modulation is the only option. Minimum shift keying (MSK) was chosen, which is a special case of frequency shift keying (FSK), because it has better power spectrum properties compared to other digital modulation techniques such as amplitude shift keying or phase shift keying. Specifically, MSK has a power spectrum $S_{\mathrm{MSK}}(f)$ that drops as the fourth power of frequency [15]:

$$
S_{\mathrm{MSK}}(f) \approx \frac{1}{(5 T f)^{4}}
$$


as opposed to BPSK, where power spectrum drops as the second power of frequency [15]:

$$
S_{\mathrm{BPSK}}(f) \approx \frac{1}{(2 \pi T f)^{2}},
$$

where $T$ is the symbol (bit) duration. This is a useful property when two different sensors operate in closely adjacent subcarrier frequencies. In that case, utilization of MSK is preferable over BPSK (or ASK) simply because interference from one sensor to the other is minimized. ${ }^{2}$

Consecutively, $b_{i}(t)$ is written as a square wave of angular frequency $\omega_{\mathrm{s} i}$, with angle modulation represented by $\phi_{\mathrm{s} i}(t)$, in terms of its Fourier components:

$$
b_{i}(t)=\frac{4}{\pi} \sum_{k=0}^{+\infty} \frac{1}{2 k+1} \cos \left[(2 k+1)\left(\omega_{\mathrm{s} i} t+\phi_{\mathrm{s} i}(t)\right)\right] .
$$

With FSK modulation and no baseband filtering (due to the simple backscattering operation of each sensor), $\phi_{\mathrm{s} i}(t)$ can be written as:

$$
\phi_{\mathrm{s} i}(t)=2 \pi \Delta f_{s} \int_{0}^{t} \sum_{k} \mathcal{B}_{i}[k] p(\tau-k T) d \tau
$$

where $T$ is the bit duration, $\mathcal{B}_{i}[k]= \pm 1$ is the information bit pattern of sensor $i, p(t)$ is a rectangular pulse of duration $T$ and amplitude 1 and finally, $\Delta f_{s}$ is the frequency deviation. For MSK specifically, $\Delta f_{s}=0.25 / T$ [15].

The use of rectangular pulses (according to eq. (12) and $\left.\Delta f_{s}=0.25 / T\right)$ simplified practical implementation of MSK modulation at each sensor. Nevertheless, rectangular pulses also imply the existence of odd harmonics. In order to avoid interference of third (or higher) harmonics of one sensor subcarrier to other sensor signals, the minimum sensor subcarrier frequency utilized, should be chosen appropriately:

$$
3 f_{\text {sMIN }}>f_{\text {sMAX }} \Rightarrow f_{\text {sMIN }}>\frac{f_{\text {sMAX }}}{3} \text {. }
$$

The sensors and receiver built adhere to the above rule, with $f_{\text {sMAX }}=200 \mathrm{kHz}$ and $f_{\text {sMIN }}=67 \mathrm{kHz}$.

\section{The Hub Receiver RF Front-End}

The RF front-end of the hub receiver implemented in this work, only sees the fundamental component of the square wave $b_{i}(t)$. This is done in order to simplify hub receiver design, as capturing higher harmonics would need a much wider bandwidth than $B=f_{\mathrm{sMAX}}-f_{\mathrm{sMIN}}$. From (11), it can be seen that the fundamental component holds $\approx 80 \%$ of the total power of the square wave. Therefore, including all the harmonics would, at best, improve signal strength by about 1 $\mathrm{dB}$. This does not justify the substantial additional cost and complexity associated with the wider bandwidth.

Substituting the fundamental term from (11) into (8), the following expression for the backscatter signal from sensor $i$ is obtained:

$$
r_{\text {hub }}^{i}(t)=\frac{4}{\pi} A_{i}(t) \exp \left(j \omega_{c} t+\phi_{0}(t)\right) \cos \left(\omega_{\mathrm{si}} t+\phi_{\mathrm{si}}(t)\right)
$$

\footnotetext{
${ }^{2}$ Sensor subcarrier frequency allocation and relevant issues are discussed in detail in the subsection II-D.
}

It is common in backscatter communication to use homodyne detection in the receiver. This is particularly effective because the receiver is co-located with the source of the RF carrier and, by using the transmitted signal itself as a reference for homodyne detection, phase noise cancels out ([16], pp. 129-138). For the purposes of this document, it is sufficient to note that a homodyne receiver removes the RF carrier (i.e., the signal is frequency-shifted to $0-\mathrm{Hz}$ center frequency) and extracts the real and imaginary part of the received signal (also referred to as the "in-phase" and "quadrature" components). Thus, the output of the RF homodyne front-end is a pair of real signals for sensor $i$ :

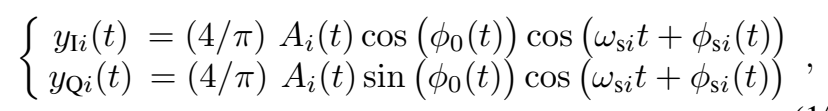

where $y_{\mathrm{I} i}(t)$ is the real part of (14) (the "in-phase" component) and $y_{\mathrm{Q} i}(t)$ is the imaginary part of (14) (the "quadrature" component).

Finally, taking into account backscattered signals from $N$ sensors, as well as noise at the receiver, the aggregate signal seen from the analog-to-digital converter (ADC) immediately after the heterodyne front-end (Fig. 2), becomes:

$$
r_{\mathrm{I}}(t)=\sum_{i=1}^{N} y_{\mathrm{I} i}(t)+n_{\mathrm{I}}(t), r_{\mathrm{Q}}(t)=\sum_{i=1}^{N} y_{\mathrm{Q} i}(t)+n_{\mathrm{Q}}(t),
$$

where $n_{\mathrm{I}}(t), n_{\mathrm{Q}}(t)$ represent additive receiver noise. Sources of receiver noise include thermal and quantization noise and the two noise components above are assumed to be lowpass, independent and identically distributed Gaussian random processes. The power spectral density of the noise is assumed flat, up to a cutoff frequency $W$, which denotes the homodyne $\mathrm{RF}$ front-end bandwidth. Their variance $\sigma^{2}$ is related to power spectral density $\mathcal{N}_{0}$ by:

$$
\sigma^{2}=\mathbb{E}\left\{n_{\mathrm{I}}^{2}\right\}=\mathbb{E}\left\{n_{\mathrm{Q}}^{2}\right\}=W \mathcal{N}_{0} .
$$

The implemented homodyne RF front-end of this work has bandwidth $W \approx 220 \mathrm{kHz}$, accommodating the selected maximum subcarrier frequency $f_{\mathrm{sMAX}}=200 \mathrm{kHz}$ and thus, the whole sensor subcarrier frequency range.

\section{The Sensors Access}

Each sensor bit rate of $R=1 / T=10 \mathrm{bps}$ is orders of magnitude smaller than each sensor subcarrier frequency $f_{\mathrm{s} i} \in B=[67 \mathrm{kHz}, 200 \mathrm{kHz}]$. Therefore, eqs. (15) imply that each sensor's signal is a narrow-band signal centered around its own and unique subcarrier frequency, allowing multiple sensors to operate simultaneously in this subcarrier frequency range. Fig. 3 provides a snapshot of the received backscattered signal spectrum, ${ }^{3}$ around the carrier frequency $f_{c}$. Notice that RF clutter around the carrier frequency is typical, due to scatterers around the hub, and that is why $f_{\text {sMIN }}>>0 \mathrm{~Hz}$.

The continuous operation of all sensors differentiates this work from traditional RFID systems, where usually the interrogator access a subset of the tags (sensors) within a

\footnotetext{
${ }^{3}$ This is the received signal at the hub receiver ( $\left.\mathrm{Rx}\right)$ antenna, before heterodyne downconversion to dc.
} 
TABLE I

Simulation Parameters for Edge Collision Probability EVALUATION

\begin{tabular}{|r|l|}
\hline$R$ & $10 \mathrm{bps}$ \\
$d_{\max }$ & $100 \mathrm{~m}$ \\
$d_{\min }$ & $10 \mathrm{~m}$ \\
$P_{\mathrm{T}}$ & $30 \mathrm{dBm}$ \\
$f_{c}$ & $900 \mathrm{MHz}$ \\
$G_{\mathrm{T}}$ & $9 \mathrm{~dB}$ \\
$G_{\mathrm{R}}$ & $3 \mathrm{~dB}$ \\
$h_{\mathrm{T}}$ & $3 \mathrm{~m}$ \\
$h_{\mathrm{R}}$ & $0.25 \mathrm{~m}$ \\
$\eta$ & $-10 \mathrm{~dB}$ \\
$\mathcal{N}_{0}$ & $-164 \mathrm{dBm} / \mathrm{Hz}$ \\
\hline
\end{tabular}

percentage of time (not continuously). The latter scenario occurs when a) a single tag passes within working range of the interrogator [8], as in toll collection scenarios or b) tags are equipped with receivers and can be addressed individually [17], or in groups with the help of a tree-based anti-collision algorithm (e.g. see [11]). The sensor access scheme in this work is, in principle, based on the limited required bandwidth for each sensor and the fact that different sensors operate in different subcarrier frequencies, in conjunction with a flexible data processor (discussed subsequently).

In practice, given that sensors continuously modulate without any baseband filtering mechanism (due to backscatter operation), adjacent in subcarrier frequency sensors might interfere with each other. This event might occur depending on how close in subcarrier frequency are the sensors and how much different is their signal power. Following the notation of this work and particularly eqs. (7), (9), signal-to-interferenceand-noise ratio (SINR) can be calculated, as well as the probability of SINR to fall below a target ratio (TR) for $E_{b} / \mathcal{N}_{0}$, required for successful reception:

$$
\operatorname{Pr}\left\{\frac{P_{i}^{\text {hub }} / R}{\mathcal{N}_{0}+\sum_{j \neq i, j=1}^{N} S_{\mathrm{MSK}}\left(\left|f_{\mathrm{s} j}-f_{\mathrm{s} i}\right|\right) P_{j}^{\mathrm{hub}} / R}<\mathrm{TR}\right\},
$$

where $E_{b}$ denotes energy-per-bit. Notice that interference from one sensor to another does not depend on the wireless environment between them, but instead, depends on the wireless environment (and distance) between each sensor and the hub. This is another distinctive characteristic of backscatter radio due to its two-way (from hub to sensor and back to hub), round-trip nature.

Fig. 4 plots the above probability of outage (or sensor collision, as commonly referred to in the RFID literature), for the worst-case scenario of sensor $i$ located at the edge of coverage $d_{i}=d_{\max }=100$ meters. The rest of the sensors are uniformly distributed with ranges between 10-100 meters and subcarrier frequencies randomly (i.e. not carefully) assigned. The list of parameters used are summarized in Table I. It is shown that for a moderate target ratio (TR) $E_{b} / \mathcal{N}_{0}$ of $6 \mathrm{~dB}$, the worst-case collision event occurs with probability of $\sim$ $2.5 \%$ for 100 sensors or $8.5 \%$ for 300 sensors, when sensor subcarrier frequencies are randomly assigned. We note that this is worst case scenario values, as a sensor located closer to the hub (and not at the edge of coverage), has stronger backscattered signal. Still, these numbers indicate that a rather

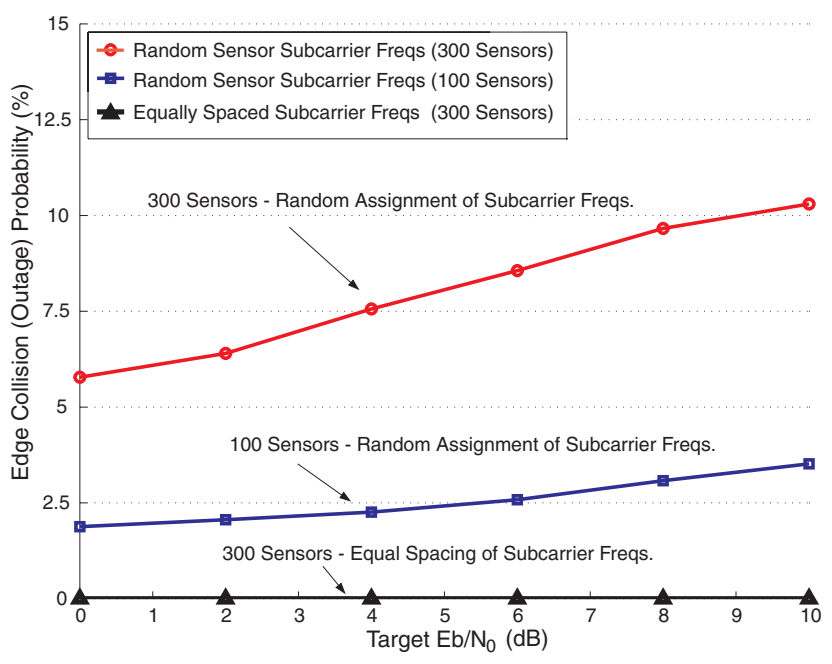

Fig. 4. Collision (outage) probability for a sensor located at the edge of coverage $(100 \mathrm{~m})$.

limited number of sensors will not work (collide). This can be compensated by installing additional sensors into the network, given their reduced complexity and cost.

The same plot shows that when subcarrier frequencies are equally spaced in the available range $B$, the above probability goes to zero, even for 300 sensors. Between the two above scenarios (random assignment or equally spaced subcarrier frequencies), there is the alternative scenario of carefully allocating frequencies for sensors closer to the hub and randomly assigning subcarrier frequencies for all the rest.

Accordingly, sensor interference is not an issue for the majority of sensors in this system, under the aforementioned assumptions. The basic reason is the limited bandwidth needed for each sensor, which is $3-4$ orders of magnitude smaller than the available subcarrier frequency band $B$, allowing simultaneous operation of multiple sensors. A similar result was also reported in [18].

Thereinafter, we strictly focus on sensors that do not collide and as such, any reference to noise will be limited to receiver noise. From eq. (15), the average energy per bit can be derived:

$$
E_{\mathrm{b}}=\left(16 / \pi^{2}\right) A_{i}^{2} T / 2,
$$

and the SNR for sensor $i$ information signal becomes:

$$
\rho=\frac{E_{\mathrm{b}}}{\mathcal{N}_{0}}=\frac{8}{\pi^{2}} \frac{A_{i}^{2} T}{\sigma^{2} / W} .
$$

The formula above assumes coherent (optimal) combining of the in-phase and quadrature components in eq. (15), as well as stable signal amplitude $A_{i}(t){ }^{4}$

\section{E. System Prototype Details}

1) $H u b$ : The frequency of the transmitted RF carrier is tunable in the range $900-930 \mathrm{MHz}$, with transmitted power at $5 \mathrm{~mW}$. A portion of the signal transmitted by the antenna is also used as the local oscillator (LO) for homodyne reception and down-conversion. As already mentioned, the bandwidth of the implemented RF front-end is $W \approx 220 \mathrm{kHz}$.

\footnotetext{
${ }^{4} A_{i}(t)=A_{i}=$ constant, otherwise $\rho$ becomes a function of time.
} 


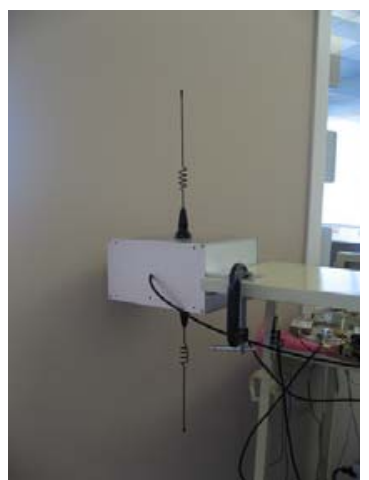

(a) Hub

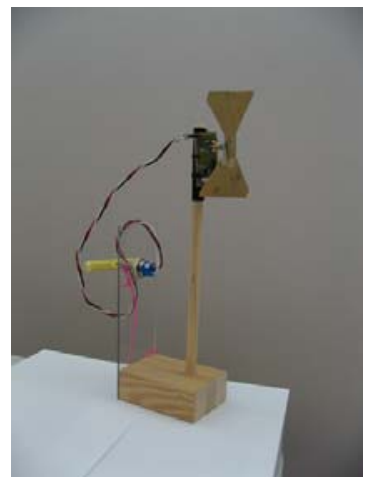

(b) Sensor
Fig. 5. The hub includes a transmit $(\mathrm{Tx})$ and a receive $(\mathrm{Rx})$ antenna. Each sensor built in this work consists of a bow-tie antenna connected to a transistor, controlled by a low-cost micro-controller and a large, manual switch (for demonstration purposes).

The result of the down-conversion is a pair of in-phase (I) and quadrature $(\mathrm{Q})$ baseband waveforms, digitized by a dualchannel 16-bit analog-to-digital converter (ADC) (Strategic Test, Ultra-Fast series). The sampling rate is $1 \mathrm{MHz}$ for each waveform (Figs. 2, 6). With this sampling rate, the Nyquist frequency is $500 \mathrm{kHz}$ which allows subcarrier frequencies up to $f_{\text {sMAX }}=200 \mathrm{kHz}$ to be accurately recorded with enough margin for digital filtering of adjacent signals. The 16-bit resolution allows adequate dynamic range to accommodate sensors at various distances from the hub. The ADC board is installed in a desktop personal computer (PC) and data processing is performed completely in software. From that perspective, the system described in this work is softwaredefined. Fig. 5(a) is a photo of the hub transmit and receive antennas. The metal box between them provides a simple, yet efficient, separation between transmit and receive RF signals.

2) Sensors: Each sensor is designed around a low-power micro-controller (Texas Instruments MSP430) driving a lowpower RF switch. The micro-controller clock is derived from a low-cost watch-type crystal at $32768 \mathrm{~Hz}$, with an accuracy of $100 \mathrm{ppm}$. The specific subcarrier frequency for each sensor, in the aforementioned range $B=[67 \mathrm{kHz}, 200 \mathrm{kHz}]$, is produced by a software-based phase-locked-loop (PLL), which makes FSK or MSK modulation easy to implement.

Each sensor is battery-assisted and operates semi-actively: battery is only used for switching on/off a single transistor, performing subcarrier modulation, without any further signal conditioning, amplification or processing. Decoupling the power requirements of each sensor to function from the power required for successful communication allows backscatter radio operation in extended ranges. Furthermore, batteries in sensors are commonly already available to power the electronics required for monitoring a specific parameter of interest (eg. pollutant concentration, humidity etc). Fig. 5(b) is a photo of one of the sensors built for experimentation, where each sensor's bow-tie antenna can be seen. Bow-tie antennas are easy to tune in a wide spectrum range around $f_{c}=900 \mathrm{MHz}$.

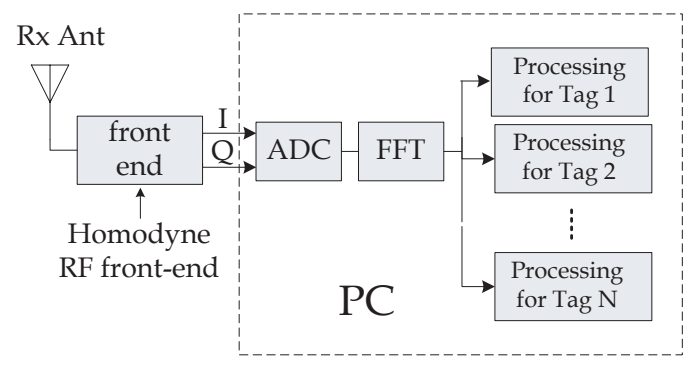

Fig. 6. The hub receiver architecture. In-phase (I) and quadrature (Q) component of the aggregate received signal is sampled and converted to the frequency domain. Processing for each individual sensor follows using a general purpose desktop computer.

\section{Data Processing}

Data processing involves the software-defined part of the receiver, which consists of a dual-channel $\mathrm{ADC}$ and a desktop PC. The received signal after homodyne RF processing (eq. (16)) is sampled and processed in software, with the following goals:

1) to extract the useful narrow-band signals, around the subcarrier frequencies of all sensors,

2) to reduce the sampling rate appropriately, from $1 \mathrm{MHz}$ to a few tens of $\mathrm{Hz}$, given that memory and processing time per sensor signal are practically limited, especially when a large number of sensors is utilized, and

3) to detect the transmitted bit patterns and synchronize to the information bits for each sensor.

The following subsections describe in detail the techniques (and their rationale) followed in this work.

\section{A. Sensor Signal Acquisition}

The first required task is to identify how many sensors are operating and which are their respective subcarrier frequencies. Therefore, the average power spectrum is calculated using efficient fast fourier transforms (FFT) and then, a search is performed for all signals with bandwidth close to the nominal, of a few tens of $\mathrm{Hz}$. The frequency resolution used was on the order of $0.5 \mathrm{~Hz}$ and the algorithm provided an estimate of the subcarrier frequency $f_{\mathrm{si}}$ for each sensor $i \in\{1,2, \ldots, N\}$. For convenience, the search was practically performed around a list of predetermined frequencies, taking into account the accuracy of each sensor's crystal $( \pm 20 \mathrm{~Hz}$, for the crystal stability and subcarrier frequency range used). In future versions of this receiver, the search will be performed without the need for a predefined list of frequencies, requiring additional processing of the average power spectrum.

After the estimation of the subcarrier frequency $f_{\mathrm{s} i}$ for each sensor $i$, the software-defined receiver performs sensor signal filtering around each subcarrier frequency and at the same time, down-sampling from $1 \mathrm{MHz}$ to $100 \mathrm{~Hz}$ (Fig. 6). In that way, each sensor signal is down-converted to dc and the number of samples/sec/sensor is decreased by a factor of $10^{4}$, reducing computation burden considerably.

Filtering around each subcarrier frequency $f_{\mathrm{s} i}$ is performed using the following impulse response:

$$
h(t)= \begin{cases}\cos \left(\frac{\pi}{2 T} t\right) & ,-T \leq t \leq T \\ 0 & , \text { elsewhere }\end{cases}
$$


with equivalent frequency domain representation:

$$
H(f)=T \operatorname{sinc}\left[2 T\left(f-\frac{1}{4 T}\right)\right]+T \operatorname{sinc}\left[2 T\left(f+\frac{1}{4 T}\right)\right],
$$

where $\operatorname{sinc}(x)=\frac{\sin \pi x}{\pi x}$.

The above filter response coincides with the nominal pulse of MSK modulation. Filtering is performed at the frequency domain representation of $y_{\mathrm{I}}(t)+j y_{\mathrm{Q}}(t)$ (from eq. (16)), by exploiting efficient fast fourier transform (FFT) techniques and extracting two complex (or equivalently four real) signals for each sensor. The first complex signal is extracted after multiplication with $H\left(f-f_{\mathrm{s} i}\right)$ and thus, corresponds to the positive-frequencies signal component $y_{i+}(t)$ of sensor $i$. The second complex signal that corresponds to the negativefrequencies component $y_{i-}(t)$ is extracted in a similar way. Down-sampling is also performed during this filtering stage. Fig. 7 depicts the above steps for the acquisition of each sensor's signal:

$\left\{\begin{array}{c}r_{\mathrm{I} i}(t)=(4 / \pi) A_{i}(t) \cos \left(\phi_{0}(t)\right) \cos \left(\omega_{\mathrm{s} i} t+\phi_{\mathrm{s} i}(t)\right)+n_{\mathrm{I}}(t) \\ r_{\mathrm{Q} i}(t)=(4 / \pi) A_{i}(t) \sin \left(\phi_{0}(t)\right) \cos \left(\omega_{\mathrm{s} i} t+\phi_{\mathrm{s} i}(t)\right)+n_{\mathrm{Q}}(t)\end{array}\right.$

\section{B. Sensor Data Extraction}

As stated before, the SNR expression in (20) assumes coherent (optimal) combining of the two (complex) extracted signals $r_{\mathrm{I} i}(t)$ and $r_{\mathrm{Q} i}(t)$ for each sensor $i$. Coherent, or equivalently maximum ratio combining (MRC) in this problem, corresponds to:

$$
r_{\mathrm{opt}}^{i}(t)=\cos \left(\phi_{0}(t)\right) r_{\mathrm{I} i}(t)+\sin \left(\phi_{0}(t)\right) r_{\mathrm{Q} i}(t) .
$$

From the above equation, it can be seen that MRC combining requires estimation of the RF carrier phase $\phi_{0}$. Due to the low bit-rate of each sensor ( $R=10 \mathrm{bps}$ ), or equivalently the extended time duration of each bit $(T=1 / R=100$ msecs in this system), factors affecting $\phi_{0}$ including multipath, cannot be assumed stable enough within multiple bitperiods. Therefore, obtaining accurate estimates of $\phi_{0}$ is not feasible and coherent combining is not an option. This is another distinctive characteristic of the backscatter radio, as presented in this work.

Without the option of coherent combining, an important part of this work has been to identify combining techniques that are simple to implement, with a reasonable performance penalty compared to (ideal) coherent combining. To that end, a different way of decomposing the received signal is considered: instead of expressing the signal in terms of real and imaginary parts, it is expressed in terms of positive and negative frequency components. Accordingly, sensor $i$ sum of extracted I and Q signals from eq. (15) is equivalent to:

$$
\begin{aligned}
y_{\mathrm{I} i}(t) & +j y_{\mathrm{Q} i}(t)=\frac{2 A_{i}(t)}{\pi}\left\{\exp \left(j \phi_{0}+j \phi_{\mathrm{s} i}(t)\right) \exp \left(j \omega_{\mathrm{s} i} t\right)\right. \\
& \left.+\exp \left(j \phi_{0}-j \phi_{\mathrm{s} i}(t)\right) \exp \left(-j \omega_{\mathrm{s} i} t\right)\right\} \\
& =y_{i+}(t) \exp \left(+j \omega_{\mathrm{s} i} t\right)+y_{i-}(t) \exp \left(-j \omega_{\mathrm{s} i} t\right),
\end{aligned}
$$

with positive and negative frequency components:

$$
\left\{\begin{array}{l}
y_{i+}(t)=\frac{2 A_{i}(t)}{\pi} \exp \left[j\left(\phi_{0}(t)+\phi_{\mathrm{s} i}(t)\right)\right] \\
y_{i-}(t)=\frac{2 A_{i}(t)}{\pi} \exp \left[j\left(\phi_{0}(t)-\phi_{\mathrm{s} i}(t)\right)\right]
\end{array} .\right.
$$

This decomposition is convenient because:

1) Narrow-band filtering and signal acquisition for each sensor (described previously) is performed at the frequency domain, with FFT-based techniques. As a byproduct, positive $y_{i+}(t)$ and negative $y_{i-}(t)$ frequency components of each sensor $i$, are readily available with no additional processing.

2) It contrasts with (15), where the two components have the same sub-carrier phase, but different and unknown relative amplitudes. Here, the two components have the same amplitude but an unknown relative phase difference. Therefore, a detection scheme that is tolerant of the unknown phase difference can be used, while combining the two components with equal weights. This is not surprising because the manipulations we applied to obtain (26) from (15) are a form of coordinate rotation

3) The format of (26) suggests a (nonlinear) technique to combine the two signals, $y_{+}(t), y_{-}(t)$ as shown below.

Specifically, the multiplication of $y_{i+}(t)$ with the complex conjugate of $y_{i-}(t)$ provides:

$$
\Lambda(t)=y_{i+}(t) y_{i-}^{*}(t)=\frac{4 A_{i}^{2}(t)}{\pi^{2}} \exp \left(2 j \phi_{\mathrm{s} i}(t)\right) .
$$

It is observed that $\Lambda(t)$ is still an angle-modulated waveform, but the angle modulation is doubled and independent of $\phi_{0}(t)$. As such, the ability to eliminate the random phase shift in the RF carrier, by mixing the two sidebands, is advantageous.

Consecutively, the software-defined receiver implements in software a detector of the instantaneous frequency $\widehat{\mathcal{B}}_{i}(t)$ :

$$
\widehat{\mathcal{B}}_{i}(t)=\frac{1}{4 \pi \Delta f_{s}} \frac{d}{d t}<\Lambda(t) .
$$

where the operator $<x(t)$ denotes phase of complex signal $x(t)$. The factor of 4 (as opposed to a factor of 2 ) is due to the signal combining technique followed in (27), where phase is doubled. From eq. (28), (27) and (12), the estimated message bit sequence $\widehat{\mathcal{B}}_{i}[k]= \pm 1$ of sensor $i$ is related to $\widehat{\mathcal{B}}_{i}(t)$ as:

$$
\widehat{\mathcal{B}}_{i}(t) \propto \sum_{k} \widehat{\mathcal{B}}_{i}[k] p(t-k T),
$$

where $p(t)$ is a rectangular pulse of duration $T$ and amplitude 1 , as previously defined in (12).

Performance of the above non-linear technique in the presence of noise, was evaluated using simulations as well as experimentation with the hardware prototype. Results are presented in section III-C below.

1) Data Synchronization: Data synchronization becomes challenging, given the absence of a receiver structure at each sensor. The latter excluded the use of pilot tones that could be transmitted from the hub to signal the beginning of each sensor's information transmission.

In this work, data synchronization is performed utilizing a pseudo-random bit-pattern, a priori known at the hub receiver. The sensor is always transmitting the synchronization (sync) bit-pattern before the actual message bit-pattern. Synchronization is performed at the demodulated baseband received signal $\widehat{\mathcal{B}}_{i}(t)$ of sensor $i$, by a simple correlation with a waveform generated according to the sync bit-pattern (similarly to eq. (29)). The found peak of correlation marks the beginning of 


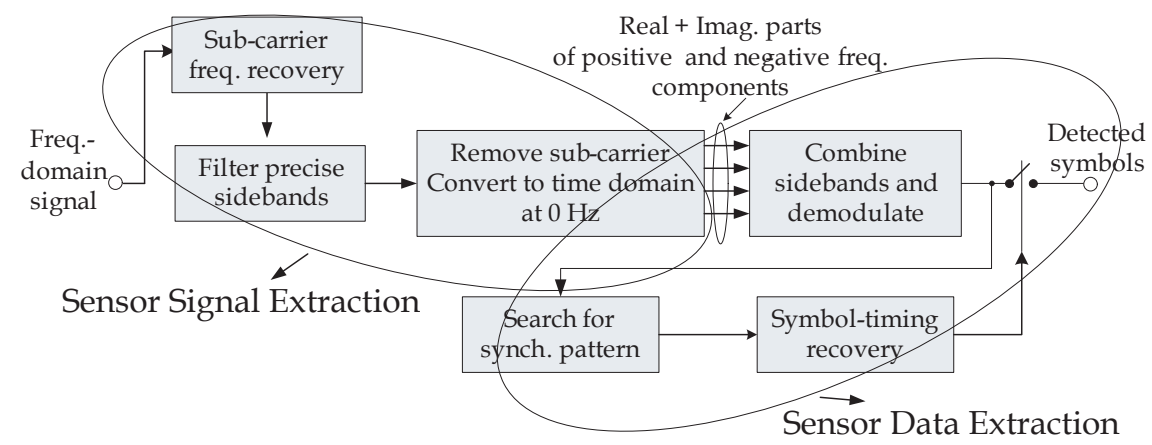

Fig. 7. The basic signal processing blocks for each sensor's information signal extraction. The receiver performs in software signal filtering, downsampling, synchronization and detection.

the sync bit-pattern and consecutively, the beginning of the message bit pattern for each sensor. We note that the sync pattern need not be unique for each sensor, but instead, a common word could be used. Nevertheless, the implemented receiver of this work utilized different sync words, treating each sync word as the unique identification number for that sensor.

An interesting question arises regarding the necessary sync word length (in number of bits), needed for minimum synchronization error. Obviously, this length depends on several parameters including bit-error-rate (BER), and consecutively, operating $E_{b} / \mathcal{N}_{0}$, as well as the length of the signal observation window, where correlation is performed. The appendix provides an answer to that question. In the reported experimental results using the hardware prototype, the length of the sync word was set sufficiently large, in order to evaluate BER without any bias from synchronization errors.

Fig. 7 depicts the steps described above, needed for each sensor's data extraction, including signal combining, detection and synchronization. ${ }^{5}$

\section{BER Results}

Performance of signal and data extraction for each sensor was evaluated with simulation, as well as experimental measurements from the actual radio prototype. The goal was to verify that end-to-end performance was not compromised during any of the processing steps described above, such as sub-carrier frequency offset estimation, data synchronization and non-linear detection.

Fig. 8 provides simulation results, assuming a sensor continuously transmitting a unique word of 128 bits followed by a message of another 128 bits. The plot includes the overall bit-error-rate of the presented receiver and contrasts it to the theoretically optimal, based on coherent detection and perfect synchronization. It is again remarked that the latter is practically infeasible given the slow bit rate in this system, which precludes any attempt for accurate carrier phase estimation, as explained before. The overall performance of our system (including synchronization as well as detection) provided an approximately $5-\mathrm{dB}$ performance difference,

\footnotetext{
${ }^{5}$ Notice that fig. 7 depicts one more module on bit duration estimation for each sensor, given that in practice, that value deviates from the nominal value of $T$. We do not discuss that module given that its contribution to the overall performance was not critical.
}

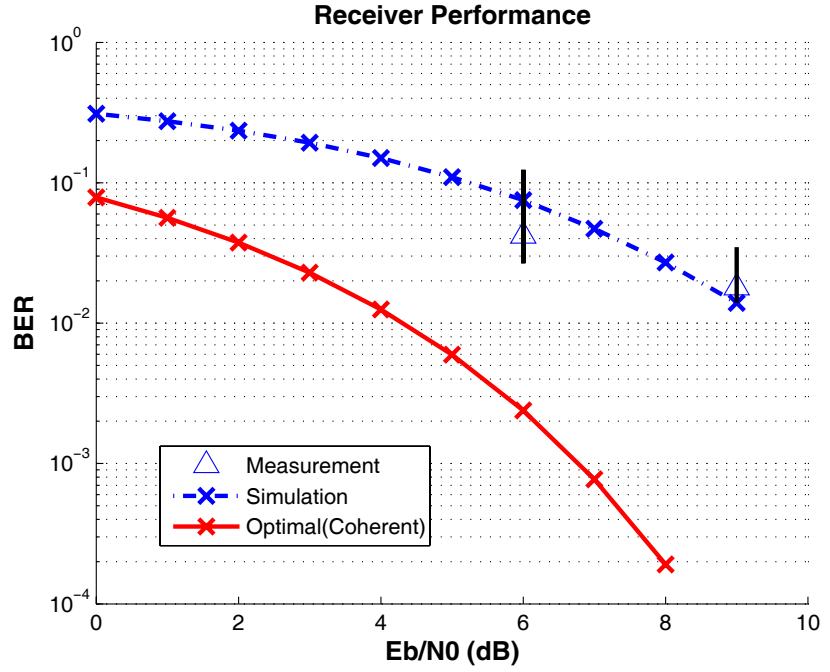

Fig. 8. The bit-error rate (BER) as a function of SNR is depicted. Performance is evaluated with simulated data, as well as with experimental data from the implemented system. In the latter case, measurement error standard deviation bars are also plotted.

compared to (theoretically optimal) coherent detection (with perfect synchronization). For a wireless backscatter link of distance $d$ between hub and sensor and assuming power loss proportional ${ }^{6}$ to $1 / d^{8}, 5 \mathrm{~dB}$ increase in signal power provides $\sim 15 \%$ increase in distance.

Fig. 8 also provides experimental results from the radio prototype, at two specific SNR values. The first is at $6 \mathrm{~dB}$ and the second is at $9 \mathrm{~dB}$, at two different locations within approximately 15 meters from the interrogator hub, in a typical indoor office space environment, with many scatterers and attenuators due to building columns, glass separators and walls. The measurements were acquired overnight when no people move in order to ensure that multi-path did not dramatically vary the received SNR. Specifically, measured data were analyzed only if the received SNR was stable enough, within $0.5 \mathrm{~dB}$. Measurement of received SNR was performed alongside eq. (20) on the experimental data. The same SNR measurement technique was double-checked in pass-band (at $1 \mathrm{Mhz}$ sampling rate) simulated backscatter

\footnotetext{
${ }^{6}$ one way propagation provides signal power loss proportionally to $1 / d^{4}$ and thus, round-trip power loss is proportional to $1 / d^{8}$.
} 
signal as well as in baseband (at $100 \mathrm{~Hz}$ ) simulated backscatter signal, where SNR was apriori known.

Fig. 8 also includes the measurement error bars, assuming a Poisson distribution for the number of bit errors. Average number of bit errors and variance were calculated by the product of BER (calculated from simulation) with total number of bits used in the experimental measurement. The reported measurement error bars have length of 2 standard deviations. It is observed that the the acquired measurements were within the error bars, demonstrating that the end-to-end radio implementation, including sensor signal filtering, downsampling, down-conversion, demodulation, synchronization and detection as described in the previous subsections, met the theoretical performance (indicated by the simulation results). The observed 5-dB performance difference compared to coherent (optimal) detection can be compensated, when the transmission power is increased to the maximum allowable level from $5 \mathrm{~mW}$ to $1 \mathrm{~W}$ (23-dB increase). Furthermore, it is noted that range will be further extended while operating outdoors, away from the RF-cluttering environment of an indoor lab.

In short, experimentation with the radio prototype provided a proof-of-concept demonstration of a working backscatter radio system, especially built for ultra low-cost sensor communication.

\section{CONCLUSION}

This work attempts to offer a new perspective to the problem of scalable, energy efficient communication in wireless sensor networks, especially when ultra low-cost sensors are required. It was demonstrated in practice that backscatter communication is a viable way for low-cost sensor networks.

In future work it is planned to optimize several modules of the system, from antenna reflection efficiency to data detection techniques for each sensor. The final goal is to install a 100meter range, 1000-sensor version of this system in a botanical garden.

\section{ACKNOWLEDGEMENTS}

The authors would like to thank research staff and administration of Mitsubishi Electric Research Laboratories (MERL) for the kind assistance and support, throughout this work. They are also grateful to the anonymous reviewers whose comments significantly improved the quality of presentation.

\section{APPENDIX \\ ESTIMATING THE SYNCHRONIZATION ERROR}

An observation window of $L+1$ bits and a sync word of bitlength $N_{\mathrm{S}}<<L$ are assumed. Without loss of generality, it is further assumed that perfect synchronization occurs at bit 0 , while erroneous synchronization occurs at bits $1,2, \ldots, L$. The constant sync word has been generated pseudo-randomly and the demodulated baseband signal is hard-quantized ( \pm 1 output values). Under these assumptions, for (perfect or erroneous) synchronization at bit $j \in\{0,1, \ldots, L\}$, with $k_{j}$ correctly identified bits (or equivalently $N_{\mathrm{S}}-k_{j}$ incorrectly identified bit), the outcome $X_{j}$ of the correlation becomes:

$$
X_{j}=k_{j}-\left(N_{\mathrm{S}}-k_{j}\right)=2 k_{j}-N_{\mathrm{S}}, k_{j} \in\left\{0,1, \ldots, N_{\mathrm{S}}\right\} .
$$

For perfect synchronization $j=0$, the probability $q_{0}$ for correctly detecting a single bit is obviously $q_{0}=1-\operatorname{BER}\left(\frac{E_{\mathrm{b}}}{\mathcal{N}_{0}}\right)$ and thus, the number of $k_{0}$ correctly detected bits in the sync word, follows the binomial distribution:

$$
\operatorname{Pr}\left(k_{0}\right)=\left(\begin{array}{c}
N_{\mathrm{S}} \\
k_{0}
\end{array}\right) q_{0}^{k_{0}}\left(1-q_{0}\right)^{N_{\mathrm{S}}-k_{0}} .
$$

For imperfect synchronization $j \in\{1,2 \ldots, L\}$, the probability $q_{j}$ for correctly detecting a single bit becomes $q_{j}=1 / 2$ and thus, the number of $k_{j}$ correctly detected bits follows the binomial distribution:

$$
\operatorname{Pr}\left(k_{j}\right)=\left(\begin{array}{c}
N_{\mathrm{S}} \\
k_{j}
\end{array}\right) \frac{1}{2^{k_{j}}} \frac{1}{2^{N_{\mathrm{S}}-k_{j}}}=\left(\begin{array}{c}
N_{\mathrm{S}} \\
k_{j}
\end{array}\right) \frac{1}{2^{N_{\mathrm{S}}}} .
$$

The synchronization error rate SyncER can now be calculated in closed-form, from eqs. (31) and (32):

$$
\begin{aligned}
\text { SyncER } & \triangleq \operatorname{Pr}\left(\max _{j \neq 0}\left\{X_{j}\right\} \geq X_{0}\right)=1-\operatorname{Pr}\left(\max _{j \neq 0}\left\{X_{j}\right\}<X_{0}\right) \\
& =1-\operatorname{Pr}\left(\max _{j \neq 0}\left\{k_{j}\right\}<k_{0}\right) \\
& =1-\sum_{k_{0}=0}^{N_{\mathrm{S}}} \operatorname{Pr}\left(\max _{j \neq 0}\left\{k_{j}\right\}<k_{0}\right) \operatorname{Pr}\left(k_{0}\right) \\
& =1-\sum_{k_{0}=0}^{N_{\mathrm{S}}}\left(\prod_{j=1}^{L} \operatorname{Pr}\left(k_{j}<k_{0}\right)\right) \operatorname{Pr}\left(k_{0}\right) \\
& =1-\sum_{k_{0}=0}^{N_{\mathrm{S}}}\left\{\prod_{j=1}^{L}\left(\sum_{0 \leq k_{j}<k_{0}} \operatorname{Pr}\left(k_{j}\right)\right)\right\} \operatorname{Pr}\left(k_{0}\right)
\end{aligned}
$$

In the implemented system, $L+1=2 N_{\mathrm{S}}+N_{\mathrm{M}}$, where $N_{\mathrm{M}}$ is the number of bits used for the message payload. The above analysis, as well as experimental evaluation showed that $64 \leq N_{\mathrm{S}} \leq 128$ essentially eliminated the synchronization error of the receiver:

Error Rate $=$ BER $(1-$ SyncER $)+\frac{1}{2}$ SyncER $\approx$ BER.

\section{REFERENCES}

[1] H. Stockman, "Communication by means of reflected power," in Proc. IRE, pp. 1196-1204, 1948.

[2] K. Finkenzeller, RFID Handbook: Fundamentals and Applications in Contactless Smart Cards and Identification, 2nd ed. New York: John Wiley and Sons, 2003.

[3] K. V. S. Rao, "An overview of backscattered radio frequency identification systems (RFID)," in Proc. Asia Pacific Microwave Conference, vol. 3. IEEE Singapore Section, 1999, pp. 746-749.

[4] M. Philipose, J. R. Smith, B. Jiang, A. Mamishev, S. Roy, and K. Sundara-Rajan, "Battery-free wireless identification and sensing," IEEE Pervasive Computing, vol. 4, pp. 37-45, Jan. 2005.

[5] J. Paradiso, K. Hsiao, and A. Benbasat, "Tangible music interfaces using passive magnetic tags," in Proc. ACM Conf. Human Factors in Computing Systems: Special Workshop on New Interfaces for Musical Expression (CHI 2001). ACM Press, 2001.

[6] V. D. Hunt, A. Puglia, and M. Puglia, A Guide to Radio Frequency Identification. John Wiley and Sons, 2007.

[7] R. Bridgelall, "Bluetooth/802.11 protocol adaptation for RFID tags," in Proc. European Wireless Conference, 2002. [Online]. Available: http://www.ing.unipi.it/ew2002/proceedings/001.pdf

[8] A. R. Koelle, S. W. Depp, and R. W. Freyman, "Short-range radiotelemetry for electronic identification, using modulated RF backscatter," Proc. IEEE, pp. 1260-1261, Aug. 1975.

[9] U. Bilstrup and P.-A. Wiberg, "An architecture comparison between a wireless sensor network and an active RFID system," in Proc. 29th Annual IEEE Intl. Conf on Local Computer Networks, 2004, pp. 583584 . 
[10] B. Zhen, M. Kobayashi, and M. Shimizu, "To Read transmitter-only RFID tags with confidence," in Proc. 15th IEEE Intl. Symp. Personal, Indoor and Mobile Radio Communications, vol. 1, 2004, pp. 396-400.

[11] J. Banks, D. Hanny, M. A. Pachano, and L. G. Thompson, RFID Applied. John Wiley and Sons, 2007.

[12] A. Bletsas, "Evaluation of Kalman filtering for network time keeping," IEEE Trans. Ultrasonics, Ferroelectrics and Frequency Control, vol. 52, no. 9, pp. 1452-1460, Sept. 2005.

[13] W. C. Jakes, Ed., Microwave Mobile Communications. New York: IEEE Press, 1974.

[14] K. Rao, P. V. Nikitin, and S. F. Lam, "Antenna design for UHF RFID tags: a review and a practical application," IEEE Trans. Antennas Propag., vol. 52, no. 12, Dec. 2005.

[15] S. Benedetto, E. Biglieri, and V. Castellani, Digital Transmission Theory. Prentice-Hall, 1987.

[16] B. Razavi, RF Microelectronics. Prentice-Hall, 1998.

[17] J. Curty, N. Joehl, C. Dehollain, and M. J. Declercq, "Remotely powered addressable UHF RFID integrated system," IEEE J. Solid-State Circuits, vol. 40, no. 11, Nov. 2005.

[18] G. Vannucci, D. Leigh, and J. Katz, "Wide-area long-range unidirectional sensor (WALRUS) network," in Proc. IEEE International Conf. on Mobile Ad hoc and Sensor Systems, Nov. 2005.

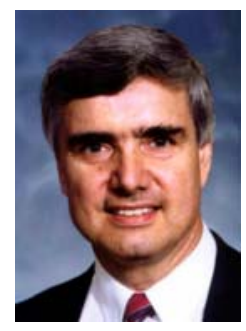

Giovanni Vannucci (S74-M79) completed a Ph.D in Electrical Engineering at Columbia University in 1979 and joined Bell Telephone Laboratories (then part of AT\&T). he remained at Bell Labs for more than two decades that saw two AT\&T divestitures and dramatic changes in communications technology. In the first few years at Bell Labs, he did research in microwave and satellite communications; later, he worked in the areas of wireless/portable communications and radiolocation. (He is co-inventor of the "Assisted GPS" location technique.) In 2000, Bell Laboratories, by now the research arm of Lucent Technologies, opened a research lab in Italy and appointed Dr. Vannucci to the post of Managing Director, which he held until his departure from Lucent in 2001. Since October 2001 he has been with Mitsubishi Electric Research Laboratories doing research in wireless networking, location techniques, RFID, and UWB communications. He has been a member of IEEE since 1974, and a senior member since 1988 .

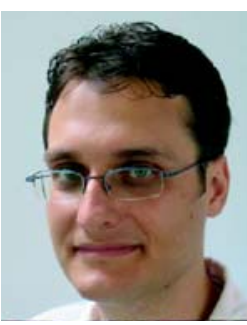

Aggelos Bletsas (S'03-M'05) received with excellence his diploma degree in Electrical and Computer Engineering from Aristotle University of Thessaloniki, Greece in 1998, and the S.M. and Ph.D. degrees from Massachusetts Institute of Technology in 2001 and 2005, respectively. He worked at Mitsubishi Electric Research Laboratories (MERL) as an intern during the summer of 2004 and as a Postdoctoral Fellow from fall of 2005 to fall of 2006, when he joined the Radio Communications Laboratory (RCL), Department of Physics, Aristotle University of Thessaloniki.

His research interests span the broad area of scalable wireless communication and networking, with emphasis on relay techniques, signal processing for communication, radio hardware/software implementations for wireless transceivers and low cost sensor networks, time/frequency metrology and nanotechnology.

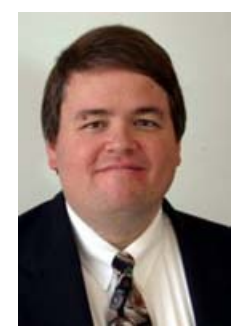

Darren Leigh earned S.B. and S.M. degrees in Electrical Engineering from the Massachusetts Institute of Technology (MIT) in 1992, and a Ph.D. in Applied Physics from Harvard University in 1998. He is currently a Senior Principle Research Scientist at Mitsubishi Electric Research Laboratories (MERL) in Cambridge, Massachusetts, where he has been on the staff for the last ten years.

His research interests range from electronic hardware and embedded systems to signal processing, $\mathrm{RF}$ and communications. Before coming to MERL, he was a researcher in radioastronomy/bioastronomy on the Harvard University/Planetary Society "Billion-channel ExtraTerrestrial Assay" (Project BETA), a search for microwave signals from extraterrestrial civilizations (SETI).

Dr. Leigh is currently working in the field of Ubiquitous/Pervasive Computing, as well as doing research on antenna optimization, phased arrays and electromagnetic simulation. 